\title{
Rapid e-mail response to first-contact e-mails increases consultation continuation rates for suicide prevention
}

\author{
Asumi Takahashi ${ }^{\mathrm{a}^{*}}$, Hajime Sueki ${ }^{\mathrm{b}}$ and Jiro Ito ${ }^{\mathrm{a}}$ \\ ${ }^{a}$ Specified Nonprofit Corporation OVA, Tokyo, Japan, ${ }^{b}$ Department of Psychology and \\ Education, Faculty of Human Sciences, WAKO University, Tokyo, Japan
}

This paper is a pre-print and has been accepted Asian Journal of Human Services Vol.20.

*Correspondence concerning this manuscript should be addressed to Asumi Takahashi. E-mail: takahashi.a@ ova-japan.org 


\title{
Rapid E-mail Response to First-Contact E-mails Increases
}

\section{Consultation Continuation Rates for Suicide Prevention}

\author{
Asumi TAKAHASHI ${ }^{1)^{*}}$ Hajime SUEKI ${ }^{2)}$ Jiro ITO ${ }^{1)}$ \\ 1) Specified Nonprofit Corporation OVA, Japan \\ 2) Faculty of Human Sciences, WAKO University, Japan
}

\begin{abstract}
Online gatekeeping is a psychological consultation service using e-mail intended for Internet users with suicide risk. One of the problems is that some users do not respond to initial contact e-mails sent. This research aimed to clarify the relation between continuation rate of the service and the reply speed to user's first-contact e-mails. 《The authors》 received initial mails from
\end{abstract} 324 people from June 2017 to June 2018, out of which 23 replies were error e-mails, and 11 people were not covered by our services; therefore, 290 users participated. We conducted a chisquare test to assess the association between three types of reply speed (within $12 \mathrm{~h}$, between 12 and $24 \mathrm{~h}$, and $24 \mathrm{~h}$ or more) and the consultation continuation rate. Reply speed for the e-mails arriving between $10 \mathrm{a} . \mathrm{m}$. and $10 \mathrm{p} . \mathrm{m}$. was related to consultation continuation: responses sent within 12 hours and those sent in over 12 hours produced continuation rates of over $70 \%$ and approximately 44\%, respectively. Help-seeking intention of users with high-risk of suicide might begin to decline after they have waited for the reply from consultants for more than 12 hours. This study contributes to effectiveness of future suicide prevention consultation using media. Systems that consultants to reply to first-contact e-mails within 12 hours are important for initiation and continuation of consultation for suicide prevention.

$<$ Key-words $>$

Suicide prevention, Online gatekeeping, E-mail consultation 


\section{Introduction}

A strategy to prevent suicide involves crisis intervention using media such as mobile phones and telephones to provide consultation activities and psychotherapy. ${ }^{1-3)}$ Internet usage rates have increased, and researchers have reported several methods of online crisis intervention. ${ }^{4-7)}$ One such technique is online gatekeeping, in which advertisements encourage individuals to use psychological consultation services when they search suicide-related words online, such as those that pertain to suicide methods. ${ }^{8)}$ This enables Internet users at high risk of suicide to access consultation services, which assess them for suicide risk and the types of issues they encounter, such as health, economic, work, and interpersonal relationships, and empowers them to seek help in the form of appropriate support.

The important advantages of online gatekeeping are twofold. First, advertisements linked to suicide-related words attract the attention of people at a higher suicide risk as opposed to those who do not search these words, and at risk individuals are then offered consultation services. ${ }^{9)}$ A previous study showed that many consultation service users were women under 30 , of whom about $80 \%$ reported suicidal ideation, about $40 \%$ reported suicide plans, and about $40 \%$ reported suicide attempts. ${ }^{7)}$ Half of them had previously visited a psychiatric hospital. Thus, getting in contact with such high-risk groups is a clear advantage. Second, e-mail consultation leads to positive psychological changes among individuals as well as help-seeking behaviors that open opportunities for appropriate support. Of course, positive psychological changes are difficult to achieve in some cases (e.g., difficulty in developing rapport with a user because of cognitive distortion or their strong mistrust of service providers), but studies have reported that about $40 \%$ of users experience psychological or behavioral changes that prevent suicide. ${ }^{7)}$ This makes online gatekeeping a promising suicide prevention technique. 
A problem related to continuous psychological consultation via e-mail for Internet users at high risk of suicide is the high dropout rate. ${ }^{8)}$ In a study on online gatekeeping, Sueki et al. replied to all first consultation e-mails from Internet users (hereafter called "first-contact e-mail/s"), but about $30 \%$ of users did not respond, and therefore, the consultation dropout rate was highest after the first-contact e-mail. ${ }^{8)}$ Online treatments for suicidal ideation have also shown that high dropout rates are a limitation. ${ }^{10,11)}$ In studies that have examined dropout rates from psychiatric services, one may assess dropout factors based on personal and medical information at the first consultation. ${ }^{12,13)}$ Meanwhile, with online gatekeeping, it is difficult to consider which personal factors are associated with dropouts from the first-contact e-mail, as users do not always divulge their personal information in these e-mails.

The discontinuation of consultation after a first-contact e-mail has been sent remains an issue, with two possible factors: the contents of the consultant's e-mail response to the user's first-contact e-mail and the urgency with which it is sent. For example, a study on suicide intervention via direct messages on social media showed that users prefer brief messages and an emphasis on their purpose. ${ }^{14)}$ Moreover, quick responses to online consultations are crucial. ${ }^{15,16)}$ In online gatekeeping practice, the contents of the reply to the first-contact e-mail have been developed based on the experience of the consultants; thus, there are not a lot of differences among the contents of the reply to the first-contact e-mail (e.g., empathetic understanding for user's story and assessment questions). On the other hand, the speed of consultants' response would likely vary depending on the consulting situation and the type of first-contact e-mails from users. Therefore, the current research aims to examine the speed of replies to first-contact emails. 
In general, mental health counseling services conducted via e-mail do not have the apparatus to respond quickly. For example, a public e-mail consultation service for the mental health of workers has specified a one-week response window. ${ }^{17)}$ E-mail consultation services at the Federation of Inochi No Denwa, which originated from Samaritans helpline organization founded in London, have stated that replies may take several days. ${ }^{18)}$ Similarly, a human rights counseling service using e-mail responds after several days. ${ }^{19)}$ Nevertheless, a study on online gatekeeping factors that can lead to consultation success has suggested replying to first-contact e-mails within a short time span. ${ }^{20)}$ This study showed no differences in consultation continuation rates for consultants' replies to first-contact e-mails within 6 or 12 hours, but the rate was lower when the consultants replied to first-contact e-mails 12 to 18 hours after receiving them. However, the study used a small sample of first-contact e-mails $(\mathrm{N}=157)$ and failed to significantly specify how quickly a consultant must respond. Therefore, the current research intends to examine differences in consultation continuation rates depending on whether an online consultant replied to a first-contact e-mail within 12 hours or more.

To achieve this purpose, we need to consider three factors associated with reply speed: (a) the day when the first-contact e-mails arrived, (b) the time when the firstcontact e-mails arrived, and (c) the textual content of the first-contact e-mails.

Regarding (a) and (b), Tirel et al. showed that 3,291 people were inclined to contact online counselors during the evening and afternoon. ${ }^{21)}$ An online counseling service for problem gambling also showed that more than half of users accessed real-time chat during the evening, overnight, or weekends. ${ }^{22)}$ Thus, there seems to be a certain range of time in which individuals use online consultation services. A study on suicide timing in Japan showed that many middle-aged males committed suicide on Monday mornings while nonworking older people often took their own lives in the morning or the early 
afternoon. ${ }^{23)}$ However, according to this study, the time of day referred to when the individual was found dead and not the time they attempted suicide. ${ }^{23)}$ Combining these factors, one may receive more first-contact e-mails in the evening and overnight.

Currently, the online gatekeeping service is not available 24 hours a day, and our staff, who work in shifts, generally respond to users between 10:00 a.m. and 10:00 p.m. In reality, however, night-shift staff sometimes reply earlier than 10:00 a.m. or later than 10:00 p.m. in emergency cases where a user is in imminent danger of dying by suicide. Nevertheless, while users can e-mail us anytime, they may not receive our responses at the time they expect. Therefore, we examine whether consultation continuation rates differ according to the interaction between the arrival time of the first-contact e-mail and the speed of response.

With regard to (c), response time also must depend on whether the first-contact email contains user consultation information. First-contact e-mails are often blank, and we have no choice but to reply with a fixed message; however, if these e-mails contain full consultation details, we need more time to read them so that we can assess the person and write a reply. The second purpose of the current research is to investigate whether consultation continuation rates differ according to the interaction between the presence of such details in the first-contact e-mail and the reply speed. 


\section{Methods}

\section{Online Gatekeeping Process}

First, when Internet users search Google for suicide-related keywords, online gatekeeping displays advertisements with headings such as "If you want to die" and "If you are thinking about how to die" on the result pages. Clicking these links sends the user to a specific website that encourages them to use psychological consultation services. This website explains the process in which a team of licensed experts (for example clinical psychologist and psychiatric social worker) conducts e-mail consultations on suicidal ideation and then provides an e-mail address for this purpose. Users can either send a first-contact e-mail to the address by themselves after clicking on "send e-mail from here" button or provide their name, address, and consultation details in the website form, which was developed by WordPress. At this point, the users were explained regarding the website, the precautions regarding the consultation, the possibility that the anonymized consultation used for research, and that as they give their consent to them, they could send this service an e-mail.

After receiving the first-contact e-mail from users in Gmail, we send them an online questionnaire to evaluate their psychological state and obtain informed consent to use their answer for this research project; we then start the gatekeeping activity. Figure 1 shows an example reply to a first-contact e-mail. Specifically, we establish rapport with users by warmly paying attention to them and showing an appreciation for the great effort they have made. We then assess their issues and evaluate their risk of suicide. The final purpose is to encourage users to seek help so that they can access more appropriate in-person support. Several studies have focused on online gatekeeping. ${ }^{6-8)}$ After directing users to face-to-face support (e.g., consultation at a psychiatric hospital near the user's residence), we follow up with them once every three to seven days for about 
one month to inquire about their current situation. The consultation concludes after we confirm the transition of support.

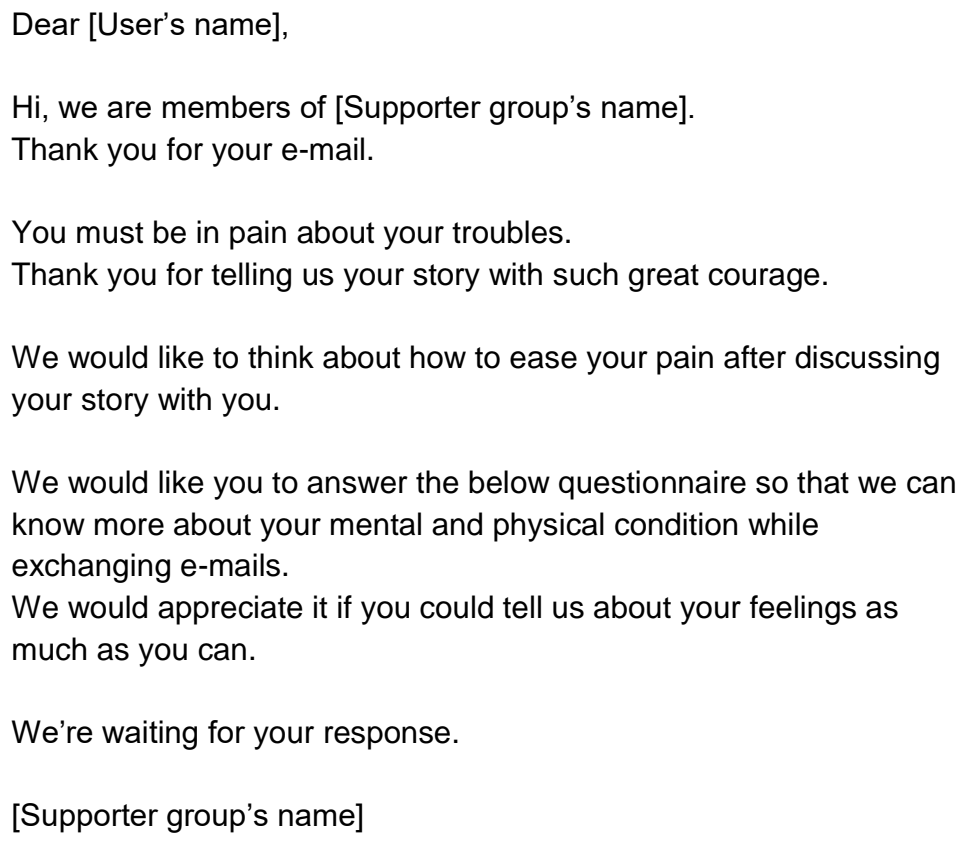

$<$ Figure 1> An Example Reply to Users' First-Contact E-mail

\section{Data Collection and Analysis}

The online gatekeeping service follows this process: A user clicks on a Google ad and sends us a first-contact e-mail, to which we respond. When the user replies, this would be considered a continuation of the consultation.

From June 2017 to June 2018, 《The authors》 conducted an online gatekeeping project for residents in the Tokyo Metropolitan Area, Japan. Google displayed advertisements that encouraged our consultation services 610,670 times with 16,587 clicks (2.72\% click rate). We received a total of 324 first-contact e-mails, which consisted of 184 from a previous study, collected from June 2017 to March 2018, and 140 new e-mails from March to June 2018. ${ }^{20)}$ 
We recorded the day and the time when we received the first-contact e-mails, whether they contain consultation details, and any user attributes that can be extracted from them. First, we used the chi-squared test to determine whether the day and time we received the first-contact e-mails and the presence of consultation information were associated with the speed of response. Second, we examined the relation between factors associated with reply speed and consultation continuation rate.

We calculated the reply speed to the first-contact e-mails by subtracting the date and time the first-contact e-mails arrived from the date and time of our reply. Although online gatekeeping prioritizes responses to first-contact e-mails, we sometimes take a long time to send our replies because of staffing issues; nevertheless, our responses rarely take more than 24 hours. To examine differences in consultation continuation rates depending on reply speed, we categorized reply speed into three: within 12 hours, between 12 and 24 hours, and after 24 hours or more.

For the e-mail arrival times, we used two shifts during which the first-contact e-mails were received - from 10:00 a.m. to 10:00 p.m. (Shift 1) and from 10:00 p.m. to 10:00 a.m. (Shift 2) —as most of our employees generally work in Shift 1 and Shift 2. The emails were also classified into two groups: blank or with text. Then, we ran chi-squared tests using HAD to analyze the relation between reply speed and consultation continuation rates based on these two categories. ${ }^{24)}$ We did not collect any personal information from the users when they clicked the advertisements or when they used the website. 


\section{Results}

\section{Information on First-Contact E-mails}

\section{1) First-contact e-mail arrival time}

Table 1 shows that most first-contact e-mails were received on Thursdays (17.6\%) and the least number of e-mails were received on Sundays (12.0\%). However, no statistically significant differences were observed from this data.

$<$ Table 1> Day and Time when the First-Contact E-mails Arrived

\begin{tabular}{cccccccc}
\hline Day & Mon & Tue & Wed & Thu & Fri & Sat & Sun \\
\hline$N$ & 44 & 46 & 45 & 57 & 49 & 44 & 39 \\
$\%$ & 13.6 & 14.2 & 13.9 & 17.6 & 15.1 & 13.6 & 12.0 \\
\hline
\end{tabular}

Figure 2 shows the arrival times of the first-contact e-mails, most of which we received at midnight. The number of e-mails showed a decreasing trend in the early morning. From 7:00 a.m. to 11:00 p.m., we received about 5 to 15 e-mails per hour. In total, 154 first-contact e-mails (47.5\%) arrived during Shift 2, and 170 first-contact emails (52.5\%) arrived during Shift 1.

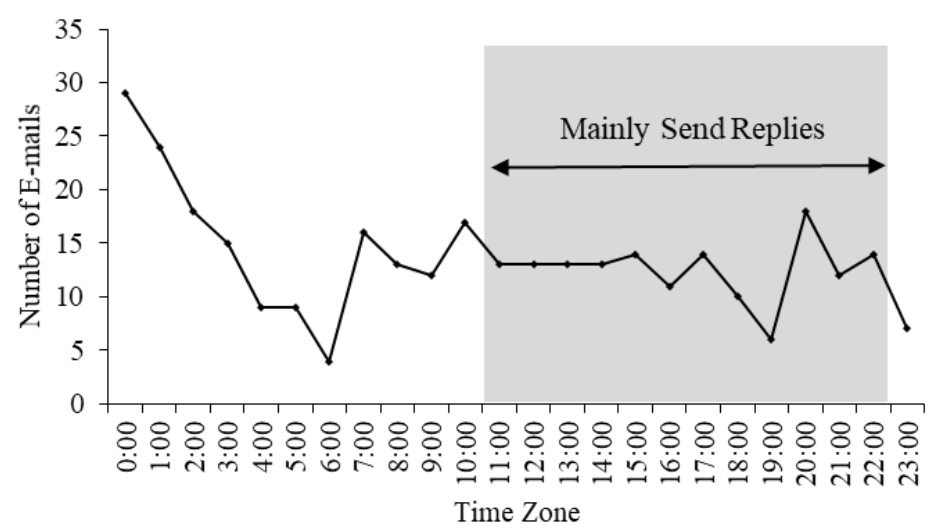

$<$ Figure 2> Time Zones of the Total Number of First-Contact E-mails that Arrived during the Survey

Period. The gray zone is the time zone in which our staff could generally send replies during Shift 1 (between 10:00 a.m. and 10:00 p.m). 


\section{2) Contents of first-contact e-mails}

Of the 324 e-mails we received, $111(34.2 \%)$ did not contain any consultation details.

\section{3) Attributes of e-mail senders}

Table 2 shows the individual characteristics (sex and age) of the e-mail senders.

Personal attributes are not often provided in the contents of the first-contact e-mails.

Judging from the number of people we could identify, many individuals were female and in their 20s and 30s. Two people stated in their e-mails that they were foreigners; however, all individuals could understand Japanese, as they search suicide-related keywords and read specific websites in Japanese.

$<$ Table 2> Analyzed Individuals who Sent First-Contact E-mails

\begin{tabular}{ccccc}
\hline & \multicolumn{4}{c}{ Responses to Our Reply } \\
\cline { 2 - 5 } & \multicolumn{2}{c}{ Yes $(n=192)$} & No $(n=98)$ \\
\hline Male & 53 & 27.60 & 8 & 8.16 \\
Female & 128 & 66.67 & 25 & 25.51 \\
Unknown & 11 & 5.73 & 65 & 66.33 \\
& & & & \\
$0-19$ & 33 & 17.19 & 9 & 9.18 \\
$20-29$ & 70 & 36.46 & 3 & 3.06 \\
$30-39$ & 37 & 19.27 & 7 & 7.14 \\
$40-49$ & 23 & 11.98 & 6 & 6.12 \\
$50-59$ & 13 & 6.77 & 0 & 0.00 \\
Unknown & 16 & 8.33 & 73 & 74.49 \\
\hline
\end{tabular}

\section{Correlation with Reply Speed}

We replied to 223 e-mails (68.8\%) within 12 hours, 54 e-mails (16.7\%) between 12 and 24 hours, and 14 e-mails (4.3\%) after 24 hours or more.

\section{1) Reply process}

Out of the 324 people who sent first-contact e-mails, we failed to deliver responses to 23 senders $(7.1 \%)$ while 11 individuals $(3.4 \%)$ were not covered by our services (e.g., they already had continuous consultations with psychiatric hospitals). Thus, we used the 
remaining 290 users to analyze consultation continuation. Among these individuals, 192 $(66.2 \%)$ responded to us at least once.

Correlation between day of the week and reply speed. No significant differences were observed between reply speed and the day the first-contact e-mails arrived $\left(\chi^{2}(12)=\right.$ $11.46, p=.49$, Cramer's V $=.14,95 \% \mathrm{CI}=.00-1.00)$.

Correlation between arrival time and reply speed. The time when the first-contact emails arrived was relevant to the speed of response $\left(\chi^{2}(2)=15.31, p<.001\right.$. Cramer's $\mathrm{V}=.23,95 \% \mathrm{CI}=.13-.35$ ), as shown in Table 3 . We replied more frequently to firstcontact e-mails arriving during Shift 2 (adjusted residual=3.31, $p=.001$ ) between 12 and 24 hours whereas for first-contact e-mails arriving during Shift 1 (adjusted residual $=-2.43, p=.02$ ), we replied more often after 24 hours or more. We found no significant differences in replies within 12 hours regardless of the time when the firstcontact e-mails arrived.

Correlation between e-mail contents and reply speed. We also observed no significant differences between reply speed and the presence of consultation content in the firstcontact e-mails $\left(\chi^{2}(2)=5.67, p=.06\right.$, Cramer's $\left.\mathrm{V}=.14,95 \% \mathrm{CI}=.00-1.00\right)$.

<Table 3> Relation between Reply Speed and Time of Users' First-Contact E-mails Arrived

\begin{tabular}{cccc}
\hline Time when Users' First-Contact & \multicolumn{3}{c}{ Reply Speed } \\
\cline { 2 - 4 } E-mails Arrived & within $12 \mathrm{~h}$ & between 12-24 h & 24 h or more \\
\hline \multirow{2}{*}{ Shift $1^{\mathrm{a}}(n=136)$} & 111 & 14 & 11 \\
& $81.6 \%$ & $10.29 \%$ & $8.1 \%$ \\
Shift $2^{\mathrm{b}}(n=154)$ & $(1.79)$ & $(-3.31)$ & $(2.43)$ \\
& 112 & 39 & 3 \\
& $72.7 \%$ & $25.3 \%$ & $2.0 \%$ \\
& $(-1.79)$ & $(3.31)$ & $(-2.43)$ \\
\hline
\end{tabular}

a Shift 1 = between 10:00 a.m. and 10:00 p.m. b Shift 2 = between 10:00 p.m. and 10:00 a.m.; first line $=$ number of e-mails; second line = percentage of total e-mails received for a given time that were replied to within the time range; third line $=$ adjusted residual. 


\section{Correlation with Consultation Continuation Rate}

Day of the week when the first-contact e-mails arrived. Table 4 shows the results of the chi-squared test. No significant associations were found between reply speed and consultation continuation rates based on the day of the week when the first-contact emails arrived (Mon: $\chi^{2}(2)=4.73, p=.09$, Cramer's V=.35; Tue: $\chi^{2}(2)=1.36, p=.51$, Cramer's V=.18; Wed: $\chi^{2}(2)=1.97, p=.37$, Cramer's V=.22; Thu: $\chi^{2}(2)=2.59, p=.27$, Cramer's V=.22; Fri: $\chi^{2}(2)=1.61, p=.45$, Cramer's V=.20; Sat: $\chi^{2}(2)=0.57, p=.75$, Cramer's V=.12; Sun: $\chi^{2}(2)=5.82, p=.05$, Cramer's V=.41; all days of the week Cramer's 95\% CI=0.0-1.0).

$<$ Table 4> Relation between Reply Speed and the Existence of Consultation Contents in Users' FirstContact E-mails

\begin{tabular}{cccc}
\hline Existence of Consultation & \multicolumn{3}{c}{ Reply Speed } \\
\cline { 2 - 4 } Contents in Users' First- & within $12 \mathrm{~h}$ & between $12-24 \mathrm{~h}$ & 24 h or more \\
\hline Contact E-mails & 146 & 42 & 7 \\
Full $(n=195)$ & 74.9 & 21.5 & 3.6 \\
& $(-1.17)$ & $(2.06)$ & $(-1.41)$ \\
Blank $(n=95)$ & 77 & 11 & 7 \\
& 81.1 & 11.6 & $(1.41)$ \\
\hline
\end{tabular}

First line $=$ number of e-mails; second line $=$ percentage of total e-mails with/without consultation contents that were replied to within the time range $(\%)$; third line $=$ adjusted residual.

Reply speed and time when the first-contact e-mails arrived. Figure 3 shows that, in the case of first-contact e-mails arriving during Shift 1, the consultation continuation rates were $72.1 \%(80 / 111)$ when we replied within 12 hours, $42.9 \%(6 / 14)$ when we replied in between 12 and 24 hours, and $45.5 \%$ (5/11) when we replied after 24 hours or more. The chi-squared test on reply speed revealed differences in continuation rates $\left(\chi^{2}(2)=7.28, p=.03\right.$, Cramer's $\left.\mathrm{V}=.23,95 \% \mathrm{CI}=0.0-1.0\right)$. Moreover, residual analysis showed that more users continued with the consultation services (adjusted residual $=2.70$, $p<.01$ ) when replied within 12 hours, but the number of users decreased (adjusted 
residual $=-2.02, p<.05)$ when we responded in between 12 and 24 hours. The continuation rate when we replied after 24 hours or more was not significant (adjusted residual $=-1.58, p=.12$ ). Among the 11 first-contact e-mails whose responses took 24 hours or more, six arrived during the holidays and the remaining e-mails arrived on weekdays.

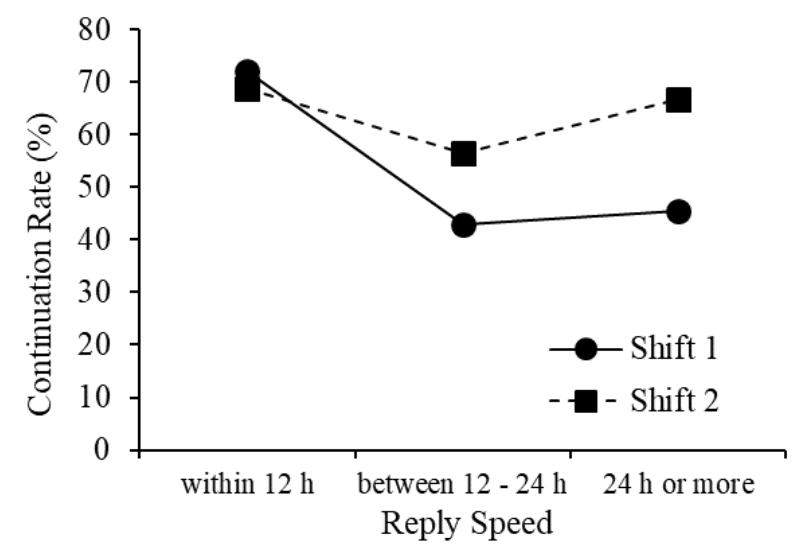

$<$ Figure 3> Relation of Consultation Continuation Rate and Reply Speed within the Time when the First-Contact E-mails Arrived. The black solid line is the continuation rate (continuation e-mails/all emails) of the first-contact e-mails arriving during Shift 1 (between 10:00 a.m. and 10:00 p.m.); the black dashed line is the continuation rate of the first-contact e-mails arriving during Shift 2 (between 10:00 p.m. and 10:00 a.m.)

For first-contact e-mails arriving during Shift 2, the consultation continuation rates were $68.8 \%$ (77/112) when we replied within 12 hours, 56.4\% (22/39) when we replied in between 12 and 24 hours, and 66.7\% (2/3) when we replied after 24 hours or more. The chi-squared test on reply speed was not significant $\left(\chi^{2}(2)=1.95, p=.38\right.$, Cramer's $\mathrm{V}=.11,95 \% \mathrm{CI}=0.0-1.0)$. Residual analysis showed no significant differences in consultation continuation when we replied within 12 hours (adjusted residual=1.35, $p=.18$ ), in between 12 and 24 hours (adjusted residual $=-1.40, p=.16$ ), and after 24 hours 
or more (adjusted residual $=-0.04, p=.97$ ). All three first-contact e-mails whose replies took 24 hours or more arrived on weekdays.

Reply speed and presence of consultation contents in the first-contact e-mails. We analyzed the relation between reply speed and consultation continuation rates based on the two categories for the contents of the first-contact e-mails. As shown in Figure 4, for first-contact e-mails with text, the consultation continuation rates were $73.3 \%(107 / 146)$ when we replied within 12 hours, 57.1\% (24/42) when we replied in between 12 and 24 hours, and $42.9 \%$ (3/7) when we replied after 24 hours or more. The chi-squared test on reply speed revealed differences in continuation rates $\left(\chi^{2}(2)=6.21, p<.05\right.$, Cramer's $\mathrm{V}=.18,95 \% \mathrm{CI}=0.0-1.0)$. Moreover, residual analysis showed that more users continued with the consultation services (adjusted residual $=2.38, p=.018$ ) when we responded within 12 hours.

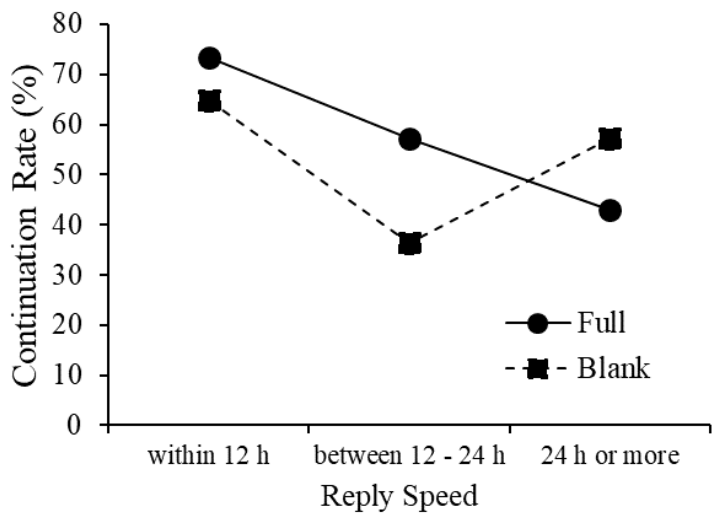

$<$ Figure 4> Relation of Consultation Continuation Rate and Reply Speed with the Presence of FirstContact E-mail Contents. The black solid line is the continuation rate (continuation e-mails/all e-mails) of first-contact e-mails with text; the black dashed line is the continuation rate of empty first-contact e-mails.

When the blank first-contact e-mails arrived, the consultation continuation rates were $64.9 \%(50 / 77)$ when we replied within 12 hours, 36.4\% (4/11) when we replied in between 12 and 24 hours, and $57.1 \%$ (4/7) when we replied after 24 hours or more. The 
chi-squared test on reply speed was not significant $\left(\chi^{2}(2)=3.35, p=.19\right.$, Cramer's $V=.19$, $95 \% \mathrm{CI}=0.0-1.0)$. Residual analysis showed no significant differences in consultation continuation when we responded within 12 hours (adjusted residual=1.61, $p=.11$ ), between 12 and 24 hours (adjusted residual $=-1.79, p=.07$ ), and after 24 hours or more (adjusted residual $=-0.22, p=.83$ ). 


\section{Discussion}

We observed that the possibility of continuing e-mail consultation services for suicide prevention increases when replies were sent within 12 hours, which supports the hypothesis. The results showed that reply speed for first-contact e-mails arriving during Shift 1 and reply speed for first-contact e-mails that contain text were related to consultation continuation rates. These results suggest that the help-seeking behaviors of some users at risk of suicide did not recur after they have waited for 12 hours to receive a response from the consultants.

The data also imply that users' expectations for urgency of response differ depending on when they sent and what they write in the first-contact e-mail. We speculate that their expectations regarding consultation diminish quickly, as they experience difficulty seeking professional assistance and face-to-face help to begin with. For example, those who send first-contact e-mails at night or in the early morning may be willing to wait longer for a reply than those who reach out during the day, as the former are likely to think that the "reply will not come soon." Similarly, users who sent blank e-mails are inclined to send e-mails as a trial with low expectations for replies in the first place, and the time to respond might not influence their help-seeking intentions. In that respect, we may have to reply more quickly to first-contact e-mails arriving during the day.

In addition, the need for consultation may have also diminished, as users' suicidal ideation and difficulties may have weakened after they sent the first-contact e-mails. Moreover, some people may have stopped consulting after reading our responses. The worst case would be that they may not reply anymore, as they may have already died by suicide. Indeed, to reduce these risks, we conclude that it is imperative that first-contact e-mails be responded to within 12 hours. 
The first-contact e-mails for the online gatekeeping service arrived whenever people were in pain and thought about suicide regardless of the day. This was different from previous research on online consultation services that were often used on weekends. ${ }^{22)}$

On the other hand, as with some studies, many first-contact e-mails arrived at midnight, with the number decreasing in the morning. ${ }^{21,22)}$ This shows that people often search suicide-related keywords and send e-mails before going to bed at night. While individuals who are aware of consultation needs may actually afford to take action on weekends, online gatekeeping prompts a consultation whenever a user searches for such statements as "I want to die," thus the high possibility that they are not yet aware of their latent consultation needs at the time.

These findings have emphasized the importance of reply speed in suicide prevention consultations, thus contributing to the literature on e-mail consultations for other issues as well as next-generation consultation systems via media such as online chat. 


\section{Conclusion and Implications}

Consultation organizations that implement outreach activities for people at risk of suicide using search-linked advertising are required to organize their staff in such a way that users receive responses to their first-contact e-mails within 12 hours. This study highlights the need for adequate staff at all times, if at all possible, rather than simply on weekends. As mentioned in the introduction, most current e-mail counseling services declare that responses would take several days. ${ }^{18,19)}$ Thus, budget allocations for suicide countermeasures would allow for more staff to be deployed and trained. To quickly reply to first-contact e-mails with consultation details, consultation organizations and the government must provide intervention skill training such as psychological assessment and improve consultants' ability to respond to first-contact e-mails within 12 hours.

This study has three limitations that require consideration. First, the present data were insufficient in determining whether users would continue consultation services if our responses took 24 hours or more, as the sample size for this category was small. Although day of the week and reply speed were not statistically related, half of the emails whose responses took 24 hours arrived on holidays, which means replies may take longer when human resources are limited, especially on holidays.

Second, because the service users' attributes were unknown in this study, other factors may be confounded. As such, we were compelled to determine their attributes and desired services from their first-contact e-mails. Therefore, these factors were not incorporated into the analysis. For example, it may have been difficult for male users to continue consultation e-mails because, hypothetically, we inadvertently took a long time to reply to them. Similarly, a user's suicidal ideation may play a role in consultation 
continuation. Van Spijker et al. showed that severe symptoms could interrupt the accomplishment of online self-care programs for suicide prevention, suggesting that severe suicidal ideation decreases one's tendency to seek help. ${ }^{11)}$ Hence, future studies that examine consultation continuation rates after a certain period has passed since obtaining information about a user's suicide risk would offer insights into the relation between suicidal ideation severity and consultation continuation.

Third, the speed of response depends on the consulting organization's business hours, which is not a random factor. In the present study, we could not completely dismiss the influence of the correlation between the time of day when a user's first-contact e-mail arrived and the time of day when we replied to them. Therefore, future research would benefit from randomizing the day and time of response to the first-contact e-mails, as this would allow for a more precise examination of its influence on consultation continuation rates.

Little evidence has been presented regarding how online consultations, including those that use e-mails, can be effectively conducted. While this research focused on the issue of reply speed to first-contact e-mails, other factors may affect the continuation of consultation as well as its success or failure. Thus, future research efforts need to analyze how users' attributes and the reception systems of online consultation can help build a consultation service system that effectively uses human/economic capital. ${ }^{25)}$ 


\section{References}

1) World Health Organization (2014) Preventing suicide: A global imperative.

URL: http://www.who.int/mental_health/suicide-prevention/world_report_2014/en/ (27, July 2020)

2) Westefeld JS. Suicide prevention and psychology: A call to action. Prof Psychol Res Pract, 2019, 50, 1-10.

3) Zalsman G, Hawton K, Wasserman D, van Heeringen K, Arensman E, Sarchiapone M et al. Suicide prevention strategies revisited: 10-year systematic review. Lancet Psychiatry, 2016, 3, 646-659.

4) Gilat I, \& Shahar G. Emotional first aid for a suicide crisis: Comparison between telephonic hotline and internet. Psychiatry, 2007, 70, 12-18.

5) Gilat I, \& Shahar G. Suicide prevention by online support groups: An action theory-based model of emotional first aid. Arch Suicide Res, 2009, 13, 52-63.

6) Sueki H. Suicide prevention using the Internet: Mini-review and a case study in online gatekeeping activity. Weaver IB. (Ed.), Suicidal ideation: Predictors, prevalence and prevention (2015). Nova Science Publishers. 85-100.

7) Sueki H, \& Ito J. Suicide prevention through online gatekeeping using search advertising techniques: A feasibility study. Crisis, 2015, 36, 267-273.

8) Sueki H, \& Ito J. Appropriate targets for search advertising as part of online gatekeeping for suicide prevention. Crisis, 2018, 39, 197-204.

9) Sueki H (2013) Can the Internet prevent suicide? University of Tokyo Press Tokyo.

10) De Jaegere E, van Landschoot R, van Heeringen K, van Spijker BAJ, Kerkhof AJFM, Mokkenstorm JK et al. The online treatment of suicidal ideation: A 
randomized controlled trial of an unguided web-based intervention. Behav Res Ther, 2019, 119, 103406. DOI: 10.1016/j.brat.2019.05.003.

11) van Spijker BA, Werner-Seidler A, Batterham PJ, Mackinnon A, Calear AL, Gosling JA et al. Effectiveness of a web-based self-help program for suicidal thinking in an Australian community sample: Randomized controlled trial. J Med Internet Res, 2018, 20, e15. DOI: 10.2196/jmir.8595.

12) Hom MA, \& Joiner TE. Predictors of treatment attrition among adult outpatients with clinically significant suicidal ideation. J Clin Psychol, 2017, 73, 88-98.

13) Morlino M, Buonocore M, Calento A, Ravel MG, \& Schiavone V. First contact with psychiatric services: Who leaves and who remains. Gen Hosp Psychiatry, 2009, 31, 367-375.

14) Tan Z, Liu X, Liu X, Cheng Q, \& Zhu T. Designing microblog direct messages to engage social media users with suicide ideation: Interview and survey study on weibo. J Med Internet Res, 2017, 19, e381. DOI: 10.2196/jmir.8729.

5) Fukuda H, Watanabe Y, Matsuda N, Oike M, Haniu T, Yokokawa H et al. An activity report of physicians of the department of general medicine, Juntendo University Faculty of Medicine, regarding the Great East Japan Earthquake. Juntendo Med, 2013, 59, 496-501.

16) Selby P, van Mierlo T, Voci SC, Parent D, \& Cunningham JA. Online social and professional support for smokers trying to quit: An exploration of first time posts from 2562 members. J Med Internet Res, 2010, 12, e34. DOI: 10.2196/jmir.1340.

17) Ministry of Health, Labor and Welfare (2020) Ear of heart, mental health portal site for working people: "Ear of heart e-mail counseling" for working people. URL: https://kokoro.mhlw.go.jp/mail-soudan/ (27, July 2020)

18) Federation of Inochi No Denwa (2016) Consultation: Question and answer. URL: https://www.inochinodenwa.org/faq.php (27, July 2020) 
19) Ministry of Justice (2020) The human rights counseling services on the Internet: How to use human rights counseling service.

URL: https://www.jinken.go.jp/soudan/PC_AD/0101_en.html (27, July 2020)

20) Ito J, Sueki H, Takahashi A, Shimizu Y, \& Nomura T. Exploring new directions in suicide countermeasures that make use of ICT. Suicide Policy Res, 2019, 2, 28-39.

21) Tirel M, Rozgonjuk D, Purre M, \& Elhai JD. When do people seek internet counseling? Exploring the temporal patterns of initial submissions to online counseling services. J Technol Hum Serv, 2020, 38, 184-202. DOI: $\underline{10.1080 / 15228835.2018 .1561348 .}$.

22) Rodda S, \& Lubman DI. Characteristics of gamblers using a national online counselling service for problem gambling. J Gambl Stud, 2014, 30, 277-289.

23) Boo J, Matsubayashi T, \& Ueda M. Diurnal variation in suicide timing by age and gender: Evidence from Japan across 41 years. J Affect Disord, 2019, 243, 366-374.

24) Shimizu H. An introduction to the statistical free software HAD: Suggestions to improve teaching, learning and practice data analysis. J Media Inf Commun, 2016, $1,59-73$

25) Sueki H. Willingness to pay for suicide prevention in Japan. Death Stud, 2016, 40, 283-289. 